This is an electronic reprint of the original article. This reprint may differ from the original in pagination and typographic detail.

Author(s): Kulju, S.; Riegger, L.; Koltay, P.; Mattila, Keijo; Hyväluoma, J.

Title: $\quad$ Fluid flow simulations meet high-speed video : Computer vision comparison of droplet dynamics

Year: $\quad 2018$

Version:

Please cite the original version:

Kulju, S., Riegger, L., Koltay, P., Mattila, K., \& Hyväluoma, J. (2018). Fluid flow simulations meet high-speed video : Computer vision comparison of droplet dynamics. Journal of Colloid and Interface Science, 522, 48-56.

https://doi.org/10.1016/j.jcis.2018.03.053

All material supplied via JYX is protected by copyright and other intellectual property rights, and duplication or sale of all or part of any of the repository collections is not permitted, except that material may be duplicated by you for your research use or educational purposes in electronic or print form. You must obtain permission for any other use. Electronic or print copies may not be offered, whether for sale or otherwise to anyone who is not an authorised user. 


\section{Accepted Manuscript}

Fluid flow simulations meet high-speed video: Computer vision comparison of droplet dynamics

S. Kulju, L. Riegger, P. Koltay, K. Mattila, J. Hyväluoma

PII:

S0021-9797(18)30301-1

DOI:

https://doi.org/10.1016/j.jcis.2018.03.053

Reference:

YJCIS 23403

To appear in:

Journal of Colloid and Interface Science

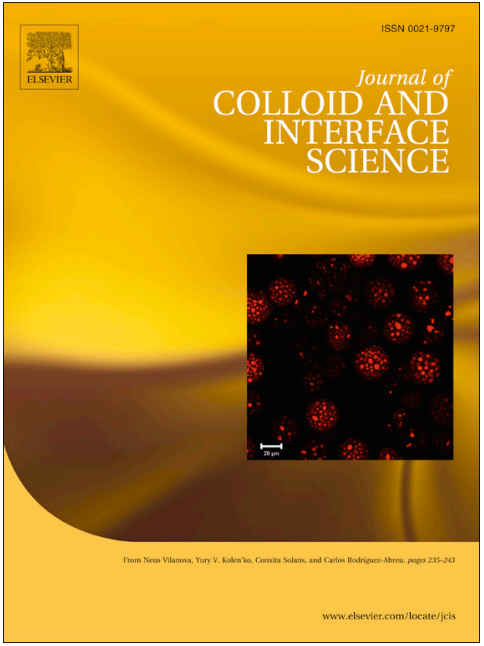

Received Date: $\quad 27$ November 2017

Accepted Date: $\quad 14$ March 2018

Please cite this article as: S. Kulju, L. Riegger, P. Koltay, K. Mattila, J. Hyväluoma, Fluid flow simulations meet high-speed video: Computer vision comparison of droplet dynamics, Journal of Colloid and Interface Science (2018), doi: https://doi.org/10.1016/j.jcis.2018.03.053

This is a PDF file of an unedited manuscript that has been accepted for publication. As a service to our customers we are providing this early version of the manuscript. The manuscript will undergo copyediting, typesetting, and review of the resulting proof before it is published in its final form. Please note that during the production process errors may be discovered which could affect the content, and all legal disclaimers that apply to the journal pertain. 


\title{
Fluid flow simulations meet high-speed video: Computer vision comparison of droplet dynamics
}

\author{
S. Kulju ${ }^{\mathrm{a}}$, L. Riegger ${ }^{\mathrm{b}}$, P. Koltay ${ }^{\mathrm{b}}$, K. Mattila ${ }^{\mathrm{c}, \mathrm{d}}$, J. Hyväluoma ${ }^{\mathrm{e}, *}$ \\ ${ }^{a}$ Natural Resources Institute Finland (Luke), Latokartanonkaari 9, 00790 Helsinki, \\ Finland \\ ${ }^{b}$ BiofluidiX GmbH, Engesserstrasse 4a, 79108 Freiburg, Germany \\ ${ }^{c}$ Faculty of Information Technology, University of Jyväskylä, P.O. Box 35 (Agora), \\ FI-40014 University of Jyväskylä, Finland \\ ${ }^{d}$ Department of Physics, Tampere University of Technology, P.O. Box 692, FI-33101 \\ Tampere, Finland \\ ${ }^{e}$ Natural Resources Institute Finland (Luke), Humppilantie 14, 31600 Jokioinen, Finland
}

\section{Abstract}

\section{Hypothesis}

While multiphase flows, particularly droplet dynamics, are ordinary in nature as well as in industrial processes, their mathematical and computational modelling continue to pose challenging research tasks - patent approaches for tackling them are yet to be found. The lack of analytical flow field solutions for non-trivial droplet dynamics hinders validation of computer simulations and, hence, their application in research problems. High-speed videos and computer vision algorithms can provide a viable approach to validate simulations directly against experiments.

\section{Experiments}

Droplets of water (or glycerol-water mixtures) impacting on both hydrophobic and superhydrophobic surfaces were imaged with a high-speed camera. The corresponding configurations were simulated using a latticeBoltzmann multiphase scheme. Video frames from experiments and simulations were compared, by means of computer vision, over entire droplet impact events.

\footnotetext{
*Corresponding author

Email address: jari.hyvaluoma@luke.fi (J. Hyväluoma )
} 


\section{Findings}

The proposed experimental validation procedure provides a detailed, dynamic one-on-one comparison of a droplet impact. The procedure relies on high-speed video recording of the experiments, computer vision, and on a software package for the analyzation routines. The procedure is able to quantitatively validate computer simulations against experiments and it is widely applicable to multiphase flow systems in general.

Keywords: lattice Boltzmann, simulation, droplet, high-speed video, experimental, hydrophobic, computer vision

\section{Introduction}

Computational fluid dynamics (CFD) is an established field with a record of successful use over decades in academic research [1] and industrial R\&D [2-4]. In fact, due to the general challenge in modelling fluid flows, CFD encompasses many specialized subfields focusing, e.g., on aerodynamics with turbulent flows, suspensions, porous media fluid flows, and multiphase fluids. Modelling the dynamics of droplets is a specific example of multiphase (or multicomponent) fluid flows. Droplet formation and dynamics are integral part of many industrial and technological processes, like spray and printing applications, along with being ubiquitous in nature, and hence already a large number of studies have concerned droplets and their dynamics [5-9]. Moreover, as a system with versatile physics involved, droplets have been also good validation benchmarks for various CFD methods [10-12].

Numerous studies concerning droplets have been conducted with the lattice Boltzmann (LB) method, including falling droplets [13, 14], droplet to droplet collisions [15] and impacts and interactions of droplets with different kind of materials [16-19], especially hydrophobic [20, 21] and superhydrophobic surfaces [22-24]. Over the years, also LB results have been validated by simulating various systems such as channel flows [25] and bubbles in liquid [26] and comparing numerical results to experimental data. In addition to those, recently the LB method has been also benchmarked in the case of droplet dynamics on surfaces [27, 28].

On the other hand, experimental validation of simulated droplet dynamics have usually been made by comparing various (system level) quantities or variables, or by observing still visualizations and images, i.e. snapshots 
from a physical incident (see e.g. Refs. [29-31]). Here our aim is to demonstrate an experimental validation procedure which captures dynamically the droplet shape and position. The direct comparisons enabled by the procedure are valuable, generally speaking, as they provide further understanding on the capabilities of computational methods (and the underlying mathematical models) including their potential in applications.

Specifically, in this study we applied LB simulations, high-speed video recording and computer vision to accomplish dynamic one-on-one comparison through the entire droplet impact event. Experiments were performed with three different liquids (deionized water and two glycerol-water mixtures) and on hydrophobic and superhydrophobic surfaces. Impacts of droplets with various features were recorded, simulated and compared. While in this article we concentrate on droplet impact on surfaces, the methodology developed and presented can in principle be applied to many other multiphase fluid systems.

\section{Methods}

Simulations were performed with the LB method. High-speed videos of experiments with droplets in flight were analyzed with computer vision to find out initial drop diameter and velocity before the impact. These values were used as input for simulations. To produce simulated high-speed video frames, three dimensional density data from simulations were visualized with isosurfaces in Mayavi [32]. Two values were visualized: the density of solid nodes (represents a hydrophobic surface in experiments) and the average of liquid and gas density in a simulation (represents a droplet). Visualizations were rendered by setting a camera in Mayavi in a way that the similar side view than in experiments was achieved. These frames were then compared to experimental video frames by a computer vision algorithm which extracts essential features like the droplet shape as well as center position from both frame types (experimental and simulated). The whole procedure to compare high-speed videos with simulations is illustrated in Fig.1. Details about the computational and experimental methods as well as computer vision algorithm are given in following sections.

\subsection{Experimental methods}

The experimental setup for generating and visualizing droplets comprises a positioning robot with integrated piezo driving electronics (BioSpot BT600, 


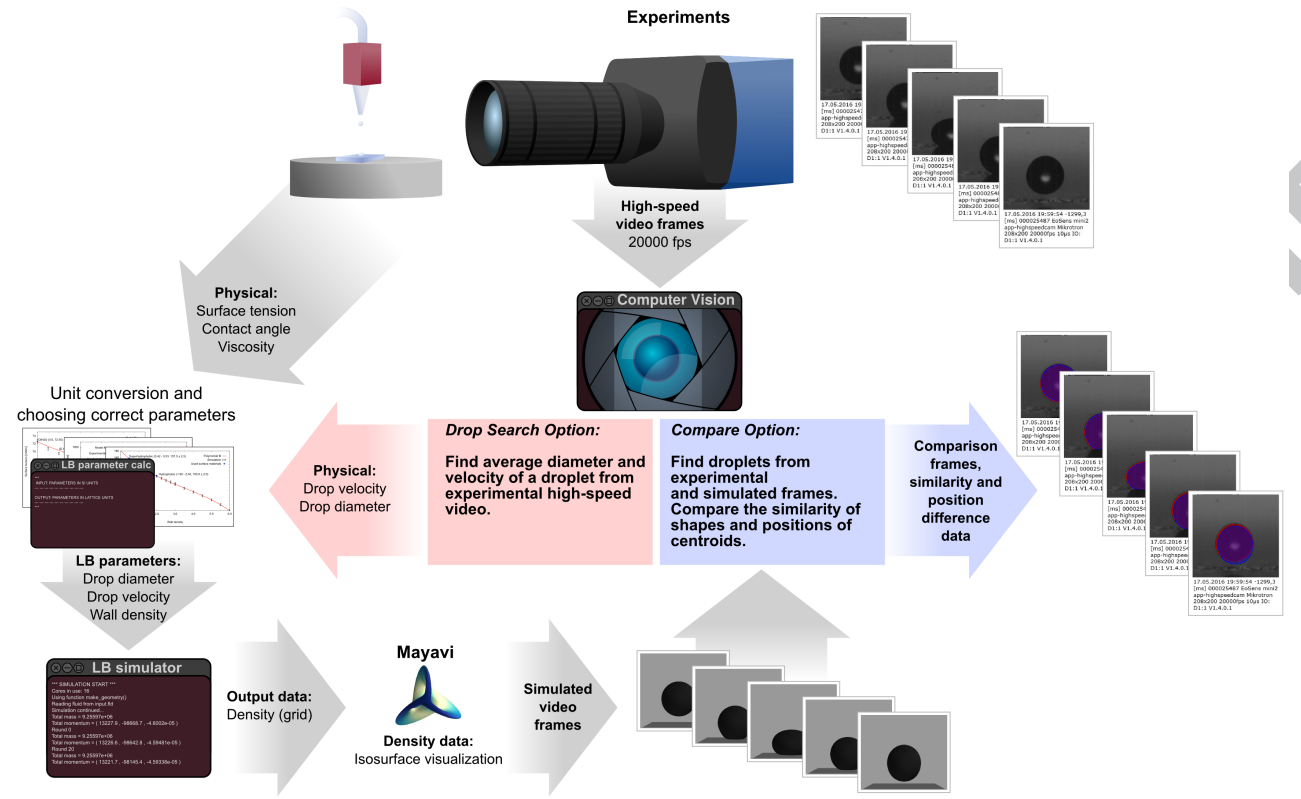

Figure 1: Procedure to compare high-speed videos with visualized frames from LB simulations.

BioFluidix, Germany), a proprietary stroboscopic system for droplet volume calibration (BioFluidix GmbH, Germany) and a high-speed camera (MotionBLITZ EoSens mini2, Mikrotron, Germany) for droplet impact visualization. The high-speed camera features a $2 \times$ magnification objective (100CAD-1640, Opto GmbH, Germany) resulting in a ratio of $2.3 \mu \mathrm{m}$ per pixel. Back lighting is realized by a LED illumination (HUEY JANN High Power LED MODULE 10W cold white) coupled with diffusor material. Two different types of droplet dispensers were utilized, one for the high $\mathrm{pL}$ range (SiJet, BioFluidix GmbH, Germany) and one for the low nL range (PipeJet P9, BioFluidix GmbH, Germany) whereas both dispensers are based on direct displacement of liquid by a piezo-stack actuator. The former generates droplets by a deflection of a silicon membrane whereas the latter generates droplets by a compression of a polymer pipe [33].

As substrates, standard microscope glass slides (Carl Roth, Germany) were used and coated with a fluoropolymer solution (Novec 1720, 3M, USA) for the hydrophobic case (static contact angle $105.0^{\circ} \pm 2.0^{\circ}$ for applied liquids) and a fluoropolymer-carbon black solution [34] for the superhydrophobic case (static contact angle $157.5^{\circ} \pm 2.5^{\circ}$ for applied liquids). For liquid 
samples, double distilled deionized water (DIH20) as well as 25 wt\% (GLY25) and 50 wt\% (GLY50) glycerol (Carl Roth, Germany) solutions were used. For hydrophobic surface contact angle hysteresis was rather high. The advancing and receding contact angles were $114^{\circ} \pm 2.2^{\circ}$ and $60.9^{\circ} \pm 1.9^{\circ}$ for distilled water and $110.6^{\circ} \pm 3.3^{\circ}$ and $53.6^{\circ} \pm 2.6^{\circ}$ for $25 \mathrm{wt} \%$ glycerol. In the first step of experiments, droplet volume respectively diameter was calibrated (i.e., $70 \mu \mathrm{m}, 140 \mu \mathrm{m}$ and $210 \mu \mathrm{m}$ ) using the stroboscopic system by varying the piezo stroke. Then, the actuation parameter reflecting kinetic energy (i.e., piezo displacement velocity) was varied. The acquired parameter combinations were then used for the high-speed imaging. After that, the respective dispenser was moved close to the substrate so that distance between a nozzle orifice and the substrate amounted to $2 \mathrm{~mm}$. Then, single droplets were dispensed onto the respective substrates and the impact of the droplet onto the substrate was recorded with an image size of $208 \times 200$ pixels and a frame rate of $20000 \mathrm{fps}$ (exposure time was set to $10 \mu$ s to reduce blurring). The experimental setup is presented in Fig. 2.

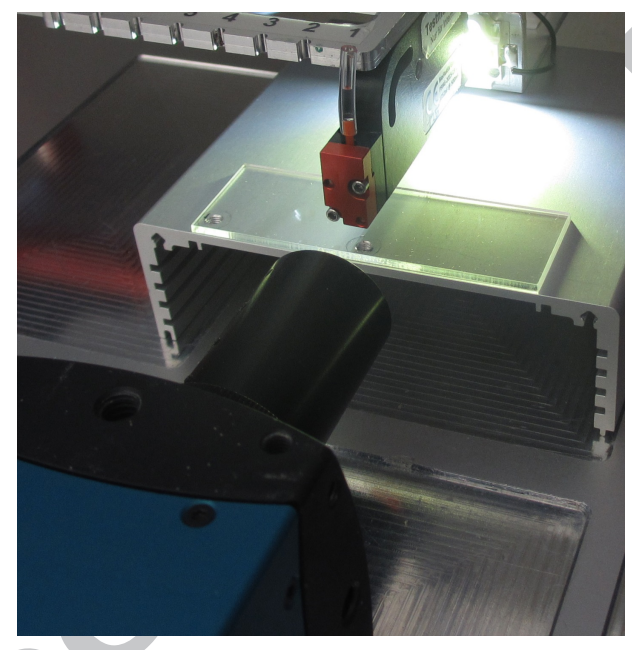

Figure 2: Closeup of experimental setup with $\mathrm{nL}$ dispenser, high-speed camera, substrate and LED lighting.

\subsection{Computational methods}

Computational simulations were performed with the LB method utilizing Shan-Chen multiphase extension [35], where the wetting behaviour was modelled by setting an effective density value for the solid nodes [36]. The 
simulation method and our implementation are described briefly in the Supplementary Material (S1.1). A more detailed account of the LB method can be found, e.g., in Refs. [37, 38].

Input parameters for LB simulation were calculated from the physical values, such as liquid material properties and the diameter and velocity of the droplet. Because impacts were recorded only by one camera, the third velocity component could not be measured. Thus during the simulations the third velocity component was set to zero. Details of material properties are presented in the Supplementary Material (S1.2).

\subsection{Computer vision and comparison}

In the following sections the two operation modes of the computer vision software and the time synchronization procedure between experimental images and the simulation results are described.

\subsubsection{Operation modes}

The computer vision application utilized in this study was coded in Python programming language. It has two operation modes:

Droplet diameter and velocity search. The software searches droplets and calculates average diameter and velocity from experimental high-speed video frames. The procedure is following:

- Experimental frames are converted to binary masks by assigning pixels darker than a given threshold color to white (1) and lighter to black (0)

- All the pixels below a given surface height are given a value 0 to remove reflections from the surface.

- Binary masks are cleaned:

- Connected components $\rightarrow$ save the largest cluster

- Noise was removed with morphological majority filter using $3 \times 3$ window (eight neighbors) and 5 / 8 majority definition (does not have to be connected)

- Reflections of the led light formed large cavities in the droplet shapes of binary masks. These were corrected by filling the black areas enclosed by a droplet shape and the surface level 
- From the acquired binary mask, the diameter and velocity of droplets are calculated, which then can be used as input values for the LB simulation.

Comparison: experiment vs. simulation. This mode compares the size, shape and position of a droplet between experimental and simulated high-speed video frames with following steps:

- Droplet binary masks are generated with same procedure that is used in the droplet search mode, but now from both frame types (i.e. experimental high-speed video as well as simulation results)

- Simulation and experimental frames are scaled and synchronized in time (see detailed description in Section 2.3.2)

- Centroids of droplets are calculated from experimental and simulation frames (binary masks)

- For shape and size comparison centroids of droplets in binary masks from experimental as well as simulation frames are placed on top of each other and a similarity index is calculated (the Jaccard similarity coefficient, as detailed in Section 2.3.3)

- For comparison of droplet dynamics, the spatial difference of centroid positions is calculated from synchronized experimental and simulation frames.

- Finally, layered comparison images are generated from synchronized experimental and simulation frames.

\subsubsection{Synchronization of experimental and simulated video frames}

Video and image sequence synchronization has been widely discussed in literature. Topics have varied from synchronization of multiple cameras filming the same scene $[39,40]$ to comparison of videos featuring similar scenes, such as human actions $[41,42]$ or magnetic resonance image (MRI) sequences [43].

In our comparison case simulated video frames could be rendered with the same camera angle as used in experiments and also the frame rate of both image sequences (experimental and simulated) were known. Moreover, simulated video frames were produced with a higher resolution and frame 
rate than experimental high-speed videos. Because of that, the accuracy of synchronization is only limited by the quality of experimental videos.

When synchronized, simulated frames are geometrically scaled (a pixel in experimental and simulated frames corresponds to the same physical length) and impact position between simulation and experiment is geometrically aligned. In addition to that, the synchronization in a time frame is performed to achieve the best matching frame set from simulated data. After synchronization, a set with the same frame rate than in the experiments can be chosen from simulated video frames for one-on-one comparison.

The first step is to calculate scaling factor which is used to resize simulated frames. This factor makes the droplet sizes in experimental and simulated frames directly comparable. Video frames from experiments and simulations are chosen at a time point before impact, where the droplets are spherical. Frames are converted to binary masks and cleaned. Droplet diameters are measured from both frames. After that, the scaling factor that is subsequently applied to all images can be calculated as

$$
F=\frac{D_{\exp }}{D_{\text {sim }}},
$$

where $D_{\text {exp }}$ and $D_{\text {sim }}$ are the diameters of droplets parsed from experimental and simulation frames, respectively.

In the following step, the best time synchronization between the experimental and simulated frames is determined. A frame with a distinctive droplet shape, usually a frame close to the moment of impact, is chosen from experimental frames as synchronization frame. Also frames before and after the chosen one are used for synchronization. This enables a better synchronization for the droplet dynamics. These frames are converted to binary masks which are then used as a comparison benchmark for simulated frames.

Frame rate of an experimental video and time steps between simulation frames are known. Thus sets consisting of three frames with same time interval as the time step between experimental frames can be formed from simulated video frames. Moving window is used to to form multiple sets as presented in Fig. 3.

Frames in a set are converted to binary masks, scaled with the scaling factor and placed to same size masks than experimental synchronization frames. After this a shift vector is calculated as a difference between positions of centroids of the droplets in centermost frames in both sets (experimental and simulation). Droplets in binary masks of simulated frames are then 


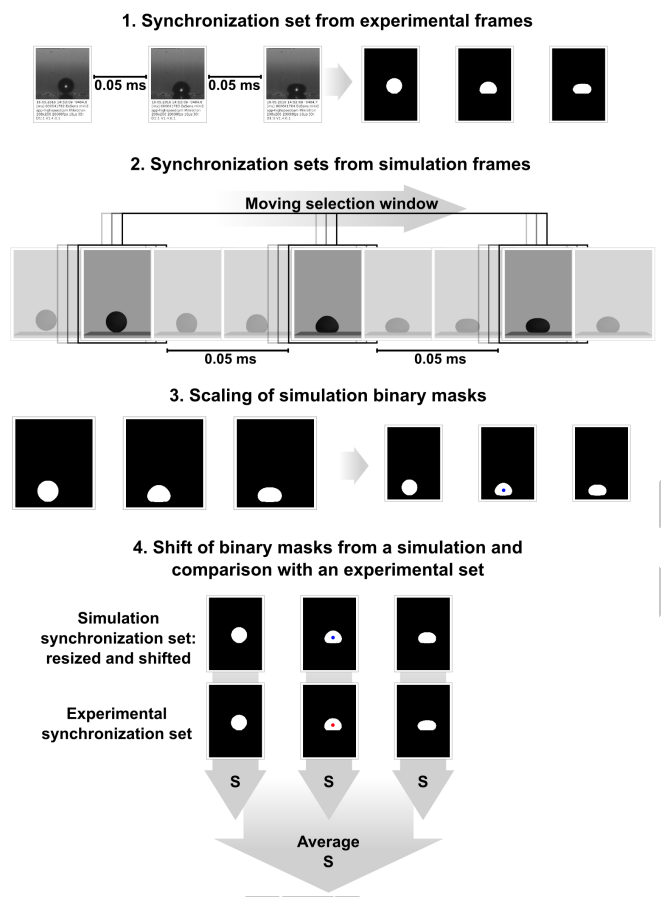

Figure 3: Procedure to synchronize experimental and simulated high-speed video frames. Centroids of droplets which are used for a shift vector calculation are marked with red and blue dots.

shifted according to this vector to generate the comparison frames. To determine the best synchronization, similarity indices (details in Section 2.3.3) are calculated by comparing the corresponding frames in experimental and simulation synchronization frame sets. A synchronization set formed from simulation frames with the best average similarity index provides the best synchronization. Because the similarity index is very sensitive to the position of compared shapes (see Supplementary Material S2.3), the method above efficiently synchronizes the time frame. The shift vector defined by this set is used when comparison frames are generated.

\subsubsection{Calculation of similarity index}

Comparison of droplet shapes from synchronized simulation video frames and experimental frames is made by calculating a similarity index. If the shift of simulation frames is the one defined during a synchronization, the similarity index measures the congruence of the shape and the position of droplets in frames. A binary mask from a simulated frame can be also shifted 
in a way that centroids of droplets (simulation and experimental) are placed on top of each other. In this case the similarity index measures just the difference of droplet shapes. For similarity index, the Jaccard similarity coefficient was chosen. It has been used to compare shapes/areas for example from MRI [44], interactive segmentation algorithms [45] and satellite images [46]. In addition to the fact that it is widely used, it is also easy to compute [46]. The index is calculated as

$$
S=\frac{\text { Drop }_{\text {exp }} \cap \text { Drop }_{\text {sim }}}{\text { Drop }_{\text {exp }} \cup \text { Drop }_{\text {sim }}},
$$

where $\operatorname{Drop}_{\text {exp }}$ and $\operatorname{Drop}_{\text {sim }}$ are the sets of the pixels of binary masks from experimental and simulated high-speed video frames. Example values for the similarity index are given in the Supplementary Material (section S2).

\section{Results and discussion}

All test cases and their details such as used liquids, surface materials, droplet diameter and velocity components are presented in Table 1. Listed droplet diameters and velocities have been calculated from experimental high-speed video frames with the computer vision algorithm described before. Diameters and velocities of droplets are average values before the impact onto the surface. The error in diameter is caused by the uncertainty of drop detection by the computer vision application as well as inevitable small variation in the droplet generation process. The velocity error is thus calculated as the standard error of weighted mean. 
Table 1: Different liquids, measured drop sizes, experimental velocities and surface materials which were considered for the benchmark. Drop diameters and velocities are average values before the impact onto a surface. Comparison videos about all the cases are presented in the Supplementary Material.

Liquids: Deionized water (DIH20), Deionized water and 25 wt\% Glycerol (GLY25), Deionized water and 50 wt\% Glycerol (GLY50)

Surface materials: Hydrophobic (H), Superhydrophobic (SH) as described before.

Physical parameters: Drop diameter $(D)$, Vertical velocity $\left(U_{V}\right)$, Horizontal velocity $\left(U_{H}\right)$, Weber number $(W e)$, Reynolds number $(R e)$

\begin{tabular}{|c|c|c|c|c|c|c|c|}
\hline Case & Liquid & Surface & $\bar{D}[\mu \mathrm{m}]$ & $U_{V}[\mathrm{~m} / \mathrm{s}]$ & $U_{H}[\mathrm{~m} / \mathrm{s}]$ & $W e$ & $R e$ \\
\hline \multicolumn{8}{|c|}{ Smaller droplets } \\
\hline 1 & DIH20 & $\mathrm{H}$ & $78 \pm 5$ & $1.29 \pm 0.04$ & $0.07 \pm 0.04$ & $1.79 \pm 0.16$ & $105.1 \pm 7.5$ \\
\hline 2 & DIH20 & $\mathrm{SH}$ & $77 \pm 5$ & $1.50 \pm 0.03$ & $0.06 \pm 0.03$ & $2.39 \pm 0.19$ & $120.6 \pm 8.2$ \\
\hline 3 & GLY25 & $\mathrm{H}$ & $67 \pm 5$ & $0.38 \pm 0.02$ & $0.00 \pm 0.02$ & $0.15 \pm 0.02$ & $11.7 \pm 1.1$ \\
\hline 4 & GLY25 & $\mathrm{SH}$ & $69 \pm 5$ & $0.40 \pm 0.02$ & $0.01 \pm 0.02$ & $0.17 \pm 0.03$ & $12.7 \pm 1.2$ \\
\hline 5 & GLY50 & $\mathrm{H}$ & $67 \pm 5$ & $0.53 \pm 0.02$ & $0.05 \pm 0.02$ & $0.31 \pm 0.04$ & $5.8 \pm 0.5$ \\
\hline 6 & GLY50 & $\mathrm{SH}$ & $71 \pm 5$ & $0.89 \pm 0.03$ & $0.08 \pm 0.03$ & $0.94 \pm 0.10$ & $10.3 \pm 0.9$ \\
\hline \multicolumn{8}{|c|}{ Larger droplets } \\
\hline $7^{*}$ & GLY25 & $\mathrm{H}$ & $215 \pm 5$ & $1.08 \pm 0.04$ & $0.16 \pm 0.04$ & $3.89 \pm 0.30$ & $107.9 \pm 4.7$ \\
\hline 8 & GLY25 & $\mathrm{H}$ & $152 \pm 5$ & $0.82 \pm 0.03$ & $0.12 \pm 0.03$ & $1.58 \pm 0.13$ & $58.0 \pm 2.9$ \\
\hline 9 & GLY25 & $\mathrm{H}$ & $146 \pm 5$ & $1.10 \pm 0.03$ & $0.15 \pm 0.03$ & $2.73 \pm 0.18$ & $74.5 \pm 3.3$ \\
\hline $10^{*}$ & GLY25 & $\mathrm{SH}$ & $213 \pm 5$ & $0.34 \pm 0.02$ & $0.05 \pm 0.02$ & $0.38 \pm 0.05$ & $33.6 \pm 2.2$ \\
\hline 11 & GLY50 & $\mathrm{H}$ & $213 \pm 5$ & $0.69 \pm 0.04$ & $0.00 \pm 0.04$ & $1.68 \pm 0.20$ & $23.8 \pm 1.5$ \\
\hline 12 & GLY50 & $\mathrm{H}$ & $214 \pm 5$ & $1.20 \pm 0.05$ & $0.00 \pm 0.05$ & $5.10 \pm 0.45$ & $41.6 \pm 2.0$ \\
\hline 13 & GLY50 & $\mathrm{H}$ & $144 \pm 5$ & $0.99 \pm 0.04$ & $0.02 \pm 0.04$ & $2.34 \pm 0.21$ & $23.1 \pm 1.3$ \\
\hline 14 & GLY50 & $\mathrm{SH}$ & $140 \pm 5$ & $0.63 \pm 0.02$ & $0.02 \pm 0.02$ & $0.92 \pm 0.07$ & $14.3 \pm 0.7$ \\
\hline $15 \dagger$ & GLY50 & $\mathrm{SH}$ & $144 \pm 5$ & $0.33 \pm 0.02$ & $0.03 \pm 0.02$ & $0.26 \pm 0.04$ & $7.7 \pm 0.6$ \\
\hline $16 \ddagger$ & GLY50 & $\mathrm{SH}$ & $143 \pm 5$ & $0.66 \pm 0.03$ & $0.03 \pm 0.03$ & $1.03 \pm 0.11$ & $15.3 \pm 0.9$ \\
\hline
\end{tabular}

$\dagger$ Sticking to the superhydrophobic surface observed, impact visualized in Fig. 6

$\ddagger$ Two droplets, cleaning of the superhydrophobic surface, impact visualized in Fig. 6 
Each test case is also characterized by two dimensionless numbers. Reynolds number describes the relative importance of inertial and viscous forces and is defined as

$$
R e=\frac{\rho U D}{\mu} .
$$

Weber number is the ratio between inertia and surface tension and reads as

$$
W e=\frac{\rho U^{2} D}{\gamma} .
$$

Above $U$ is the impact velocity, $D$ the droplet diameter and $\rho, \mu$, and $\gamma$ the density, dynamic viscosity, and surface tension of the liquid, respectively.

\subsection{Impact of a single droplet}

Cases 1 - 14 in Table 1 featured impacts of singular droplets. All these cases were simulated and analysed with computer vision. To provide examples about the dynamics of impact on both surface materials, the impact of a GLY25 droplet to the hydrophobic and superhydrophobic surface is shown in Fig. 4. Illustrated 3D droplets are rendered from simulation density data and droplet shapes parsed with the computer vision algorithm are presented below. The red area represents a droplet from an experimental frame and the blue area is a droplet from the corresponding simulated frame. The time scales of the image series are synchronized like described before. Also timestamps and shape similarity values $\mathrm{S}$ are listed in the figure.

Obviously, LB simulations are able to reproduce an impact onto a superhydrophobic surface very well. According to Fig.4 b) the shape of the droplet is congruent all the way through the impact event and the droplet is also bouncing off from the surface. However, the final velocity after the impact is sensitive to the contact angle (wall density) used in simulation. In our simulations an interval for the contact angle of superhydrophobic surface was 155 - 160 degrees and already in this interval the final velocity could be too high or too low.

Hydrophobic cases are more complex than superhydrophobic cases. The shape of the droplet is often matching experimental results accurately right after the impact. Though, when the droplet is bouncing back from the surface, droplets in simulations are often becoming too tall. This can be seen in the last frames of the visualized impact on the hydrophobic surface (Fig. 4 a). Despite the drastic bounce, the droplets in simulations are still 
a)
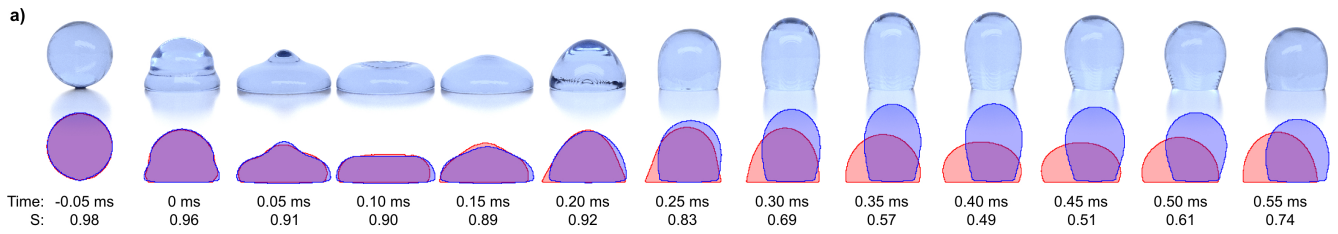

b)
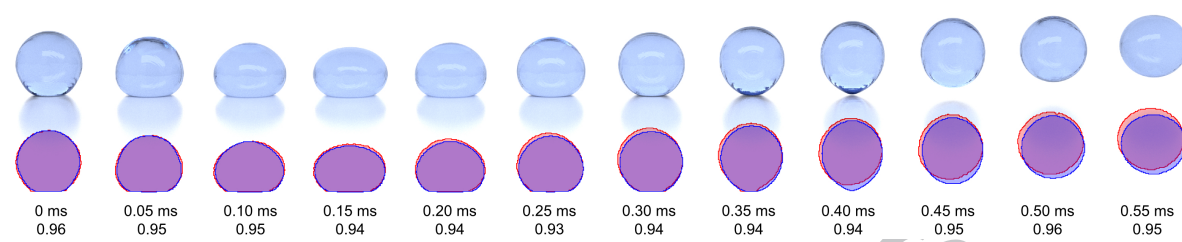

Figure 4: The impact of a GLY25 droplet to a) hydrophobic and b) superhydrophobic surfaces. 3D droplets are rendered from simulation data. Below them droplets parsed with computer vision are presented. The red area presents a droplet from an experimental frame and the blue area is a droplet from the corresponding simulated frame. Shape similarity values and timestamps are also presented below the images.

sticking to the hydrophobic surface. However, in experiments droplets stick to the point of an impact, when in simulations droplets are sliding a small distance along the surface before they attain their equilibrium shape.

The varying shape similarity in the simulations shown in Fig. 4 may be caused by the incorrect contact angle hysteresis in the simulations. In the used model there is no contact angle hysteresis for contact lines on homogeneous surfaces (see, e.g., Ref. [47]). In the simulations, the contact angle was set to measured static contact angle values, which for the considered hydrophobic surfaces is very close to the advancing contact angle but significantly different from the receding contact angle. In the first four frames shown in Fig. 4 a), which correspond to the spreading phase of the droplet impact, we can observe a very good agreement between simulation and experiment. After this, as the contact line starts to retract, simulation and experiment behave differently. Thus, as long as the contact line is advancing the correspondence is good. After the contact line starts to recede, the contact angle set for simulation no longer corresponds to the experimental case and dissimilar behavior is observed. The absence of contact angle hysteresis also explains why simulated droplets are sliding on the hydrophobic surface contrary to experiments. On the other hand, for superhydrophobic surface with negligible contact angle hysteresis correspondence between the simulation and experiments is excellent through the entire impact and rebound process. 

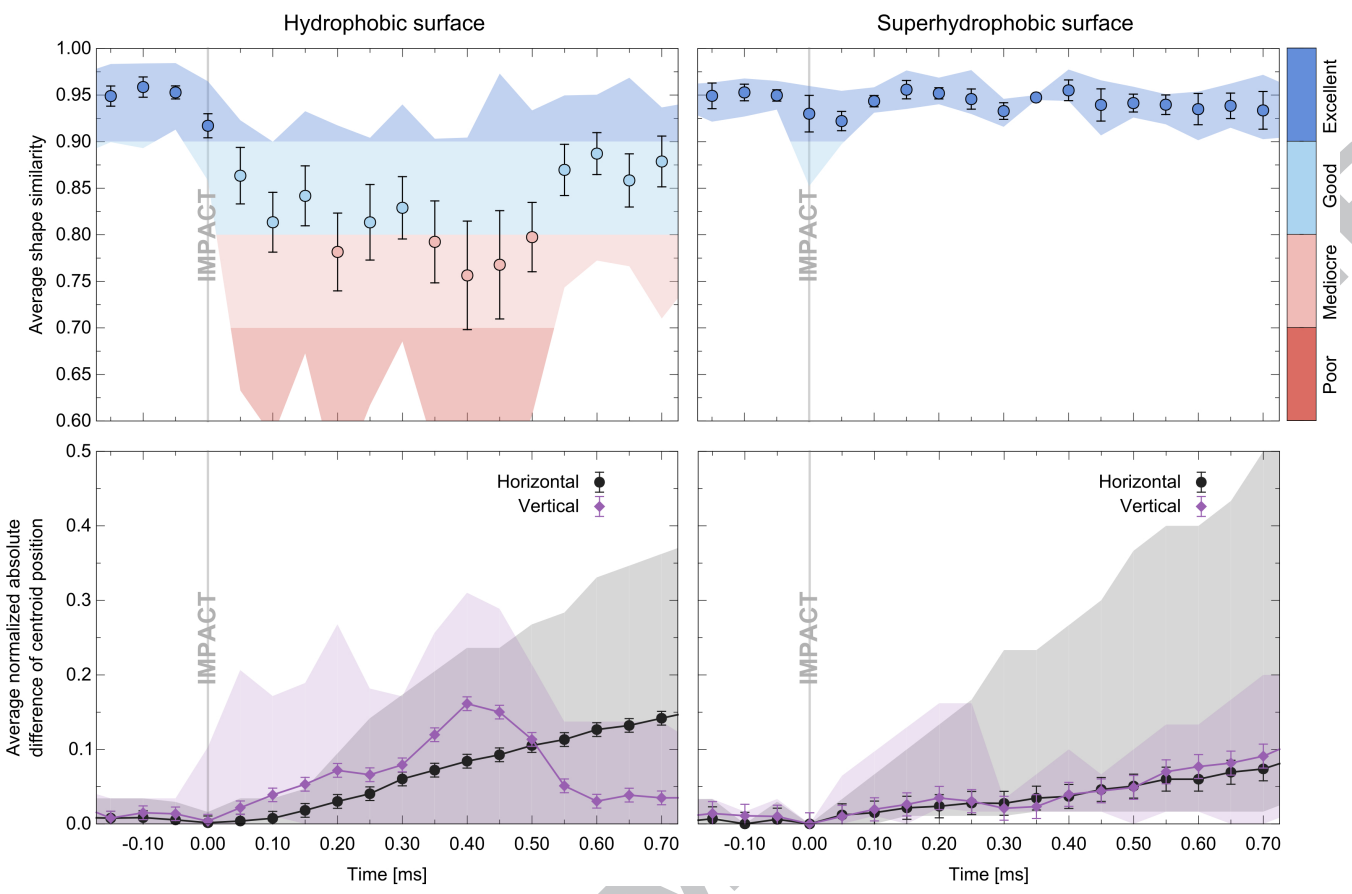

Figure 5: Average values for shape similarity and normalized absolute centroid position difference for single droplets hitting hydrophobic and superhydrophobic surface. Translucent areas in the background represent the maximum to minimum interval.

The same behaviour can be seen quantitatively in Fig. 5, where is presented average values of shape similarity and normalized droplet centroid position difference, which were calculated from all the single droplet cases. Data was collected by running computer vision comparison between synchronized experimental and simulated video frames. Shape similarity values were categorized into four different groups: Excellent $\geqslant 0.90,0.90>$ Good $\geqslant 0.80$, $0.80>$ Mediocre $\geqslant 0.70$ and $0.70>$ Poor. Because investigated cases include droplets with various sizes, the centroid position differences of droplets were normalized by dividing them with the droplet diameter.

Average shape similarity data shows clearly that droplets are congruent when the cases on superhydrophobic surfaces are considered. Shape similarity is excellent during the flight, impact and even after the bounce back. For hydrophobic surfaces, the shape similarity exhibits much higher variation, and average values after impact are dropping to Good and Mediocre categories because of too violent bounce from the surface in simulations. 
However, the shape similarity gets close to Excellent even with hydrophobic surfaces when the droplets are approaching the steady sessile state, so the simulation models the final droplet shape correctly.

When positions of a droplet centroid are inspected the average data shows the same features which were observed already from the example image series in Fig. 4. Droplets on the hydrophobic surface have large position differences in horizontal direction after impact because of the sliding effect. The vertical difference of centroid position is oscillating intensely after the impact but approaches small values when droplets are relaxing.

Droplets impacting on superhydrophobic surfaces result in average data where the difference of centroid position is linearly increasing as a function of time. The simulated droplets are flying into the correct direction but the final velocity of the droplets differs from the measured one from the experimental videos. However, the velocity differences are small, approximately 11 percent in speed and 6 degrees in direction, and the results could have been even better with a more accurate modelling of the contact angle.

\subsection{Addition and removal of a droplet from the superhydrophobic surface}

The last two cases presented in Table 1 feature special events. In case 15, a GLY50 droplet is sticking to the superhydrophobic surface. In case 16, a droplet initially in rest on the superhydrophobic surface is removed from the surface due to impact by another droplet. In the most of the videos, droplets hitting a superhydrophobic surface bounced off. However, also sticking of a droplet to superhydrophobic surface was observed when the impact velocity was low enough. Case 15 is visualized in Fig. 6 a). The droplet bounces off the surface after the impact but lands back to the surface. LB simulation was able to reproduce this effect. However, the horizontal momentum of the droplet is again conserving better in the simulation when compared to the experimental video. The same behaviour was seen also with droplets hitting the hydrophobic surface.

An attached droplet can be removed from the superhydrophobic surface when hit by a droplet having a high velocity. This effect is presented in Fig. $6 \mathrm{~b})$ where the GLY50 droplet hits and merges with the droplet attached to the surface. After the collision event the resulting droplet bounces off the surface. However, in the simulation presented in Fig. $6 \mathrm{~b}$ ) the incoming droplet had $10 \%$ higher initial velocity compared to the experiment. When the velocity recorded from the experiment was used for the incoming droplet, the merged droplet did not bounce off the surface. 

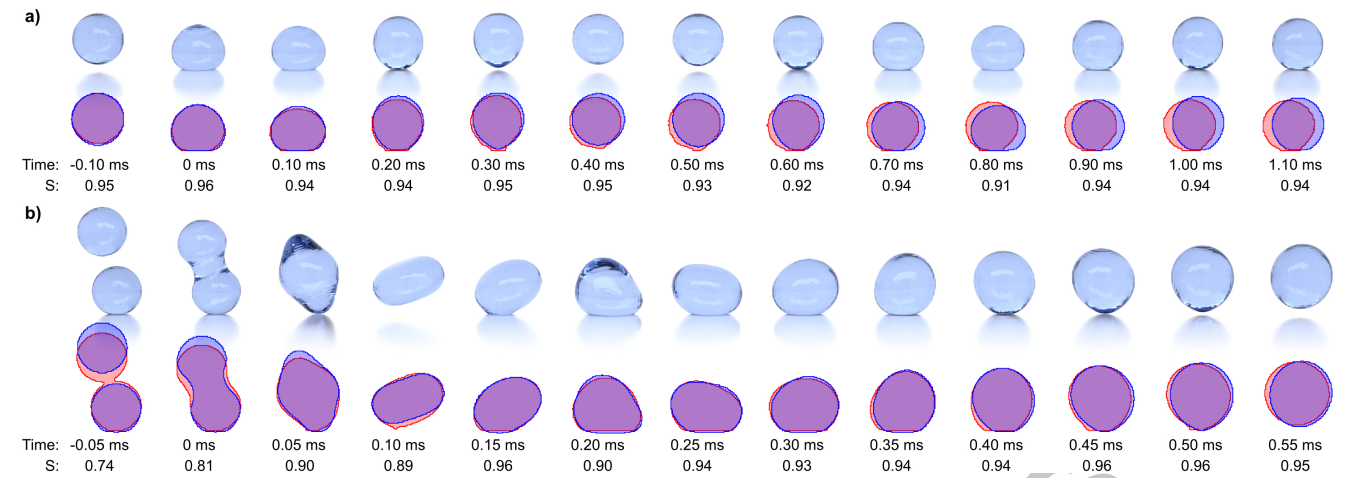

Figure 6: There are presented two cases: a) sticking of a GLY50 droplet onto the surperhydrophobic surface b) removal of a GLY50 droplet with another GLY50 droplet from the superhydrophobic surface. 3D droplets are rendered from simulation data. Below them droplets parsed with computer vision are presented. The red area represents a droplet from an experimental frame and the blue area is a droplet from the corresponding simulated frame. Shape similarity values and timestamps are also presented below the images.

As it can be seen from Fig. 6 b), the LB simulation reproduces the collision event accurately. The most interesting part about the collision is the droplet bounce off. In the experiment and simulation the initial velocity of the incoming droplet has a horizontal component pointing to viewer's right. The magnitude of this component is approximately $5 \%$ from the magnitude of the vertical component. However, when the merged final droplet bounces upwards it has horizontal momentum, but this time towards the viewer's left. The LB simulation shows also the same behaviour, so it is not related to some imperfections on the surface, but is the direct result of the droplet collision.

\subsection{Error sources}

Above we already identified some possible factors that may lead to distinction between simulations and experiments. It is likely that the imprecise modelling of the surfaces is the major error source which reduce the accuracy of the simulations. Especially, in the case of hydrophobic surfaces assumption of zero contact angle hysteresis is not realistic. Further, the results are affected by the limited resolution of the high speed videos, which cause uncertainty to the detection of a droplet centroid and diameter and this way have an influence to the selected simulation parameters. Since the experimental setup had only one camera, in simulations we had to assume 
that third impact velocity component is zero. This was not necessary true for recorded events, which may cause some discrepancy between simulations and experiments.

In addition to these error sources, additional uncertainty can be caused by some unrealistic properties of the used simulation method. The used multiphase LB model is a diffuse interface method, where the interface between the liquid and gas phases has a finite width of several lattice spacing. The effect of the diffuse interface was tested by simulating test case 7 using system size of $450^{3}$ instead of $300^{3}$ voxels which was used in the actual simulation. Corresponding droplet diameters were 204 and 132 lattice spacings, respectively. The difference between values of the shape similarity index for the two system sizes was less than 1 percent indicating that system size used in the simulations was large enough. In the test simulations, the density ratio between liquid and gas phases was 100, which is an order of magnitude lower than in experiments. The results obtained for superhydrophobic surfaces indicate, however, that the used density ratio is sufficient to obtain experimental behaviour and that viscous effects in the gas phase have minimal influence on droplet (cf. Ref. [48]).

\section{Conclusions}

In summary, we presented a validation procedure for multiphase flow simulations, which is based on high-speed video recordings and computer vision. The proposed approach enables quantitative comparison of experiments and simulations over time-dependent flow events. We utilized this procedure for detailed analysis of droplet impact on hydrophobic and superhydrophobic surfaces by comparing videos produced by high-speed imaging and dynamically similar LB simulations. Our analysis was able to identify those test cases where the used modelling approach was not able to describe the physics of the droplet-surface interaction precisely enough. The hydrophobic coating used in this study showed notable contact angle hysteresis, a property which has been observed also in previous studies with coated or native hydrophobic surfaces $[49,50]$. However, the contact angle hysteresis was not included in the model which assumed perfectly homogeneous surface. On the other hand, on superhydrophobic surface with insignificant contact angle hysteresis, videos produced by high-speed imaging and simulations were highly consistent. 
Previous studies have benchmarked LB simulations against experiments by comparing system related values, such as the contact line velocity and wet length of a droplet [27], or snapshots (still images) of physical incidents $[28,31]$. Also volume-of-fluid (VOF) based methods have been validated against experiments by comparing for example the height and diameter of a droplet during an impact $[51,52]$. In addition to numerical values, visual comparisons about droplet profiles have been presented [51, 52]. However, our method provides a dynamical one-on-one comparison about the whole impact event, providing also quantitative data about position and shape comparison. Our results show that the proposed procedure can reveal very useful information about multiphase flow processes, which can be used in validation and development of simulation methods and modelling. Furthermore, when simulations have been proved to be consistent with experiments, they can provide information which can not be extracted from experiments.

The present experimental setup had only one camera, whereby we had to assume that the velocity component in the camera direction was zero. However, the presented validation procedure can be straightforwardly generalized to two camera setup.

\section{Acknowledgments}

We would like to thank CSC-IT Center for Science (Espoo, Finland) for providing computational resources. This work was financially supported by the European Community's Seventh Framework programme NMP-2013-1.4-1 under Grant agreement No 604005 (SimPhoNy project).

\section{References}

[1] D. Greenspan, Supercomputer simulation of liquid drop formation, fall, and collision, Applied Mathematical Modelling 13 (10) (1989) 562-567. doi:10.1016/0307-904X(89)90201-1.

[2] J. Bode, Applications of computational fluid dynamics in the chemical industry, Chemical Engineering \& Technology 17 (3) (1994) 145-148. doi:10.1002/ceat.270170302.

[3] G. Scott, P. Richardson, The application of computational fluid dynamics in the food industry, Trends in Food Science and Technology 8 (4) (1997) 119-124. doi:10.1016/S0924-2244(97)01028-5. 
[4] S. T. Johansen, Multiphase flow modeling of metallurgical flows, Experimental Thermal and Fluid Science 26 (6-7) (2002) 739-745. doi:10.1016/S0894-1777(02)00183-8.

[5] H. B. Parizi, L. Rosenzweig, J. Mostaghimi, S. Chandra, T. Coyle, H. Salimi, L. Pershin, A. McDonald, C. Moreau, Numerical simulation of droplet impact on patterned surfaces, Journal of Thermal Spray Technology 16 (5-6) (2007) 713-721. doi:10.1007/s11666-007-9122-8.

[6] J. Wu, C. Liu, N. Zhao, Dynamics of falling droplets impact on a liquid film: Hybrid lattice Boltzmann simulation, Colloids and Surfaces A: Physicochemical and Engineering Aspects 472 (2015) 92-100. doi:10.1016/j.colsurfa.2015.02.045.

[7] X. Li, J. Zhao, P. Cheng, A lattice Boltzmann model for condensation and freezing of dry saturated vapor about a cryogenic spot on an inclined hydrophobic surface, International Journal of Heat and Mass Transfer 114 (2017) 628-639. doi:10.1016/j.ijheatmasstransfer.2017.06.027.

[8] D. Seo, S. Oh, S. Shin, Y. Nam, Dynamic heat transfer analysis of condensed droplets growing and coalescing on water repellent surfaces, International Journal of Heat and Mass Transfer 114 (2017) 934-943. doi:10.1016/j.ijheatmasstransfer.2017.06.129.

[9] Y. Yao, C. Li, H. Zhang, R. Yang, Modelling the impact, spreading and freezing of a water droplet on horizontal and inclined superhydrophobic cooled surfaces, Applied Surface Science 419 (2017) 52-62. doi:10.1016/j.apsusc.2017.04.085.

[10] D. J. Harvie, D. F. Fletcher, A hydrodynamic and thermodynamic simulation of droplet impacts on hot surfaces, Part I: Theoretical model, International Journal of Heat and Mass Transfer 44 (14) (2001) 26332642. doi:10.1016/S0017-9310(00)00303-3.

[11] D. J. Harvie, D. F. Fletcher, A hydrodynamic and thermodynamic simulation of droplet impacts on hot surfaces, Part II: validation and applications, International Journal of Heat and Mass Transfer 44 (14) (2001) 2643-2659. doi:10.1016/S0017-9310(00)00304-5.

[12] S. S. Sazhin, A. E. Elwardany, P. A. Krutitskii, V. Deprédurand, G. Castanet, F. Lemoine, E. M. Sazhina, M. R. Heikal, Multi-component 
droplet heating and evaporation: Numerical simulation versus experimental data, International Journal of Thermal Sciences 50 (7) (2011) 1164-1180. doi:10.1016/j.ijthermalsci.2011.02.020.

[13] X. Q. Xing, D. L. Butler, S. H. Ng, Z. Wang, S. Danyluk, C. Yang, Simulation of droplet formation and coalescence using lattice Boltzmannbased single-phase model, Journal of Colloid and Interface Science 311 (2) (2007) 609-618. doi:10.1016/j.jcis.2007.02.088.

[14] A. Fakhari, M. H. Rahimian, Simulation of falling droplet by the lattice Boltzmann method, Communications in Nonlinear Science and Numerical Simulation 14 (7) (2009) 3046-3055. doi:10.1016/j.cnsns.2008.10.017.

[15] T. Inamuro, S. Tajima, F. Ogino, Lattice Boltzmann simulation of droplet collision dynamics, International Journal of Heat and Mass Transfer 47 (21) (2004) 4649-4657. doi:10.1016/j.ijheatmasstransfer.2003.08.030.

[16] F. Varnik, P. Truman, B. Wu, P. Uhlmann, D. Raabe, M. Stamm, Wetting gradient induced separation of emulsions: A combined experimental and lattice Boltzmann computer simulation study, Physics of Fluids 20 (7). arXiv:arXiv:0910.3151v1, doi:10.1063/1.2963958.

[17] A. Dupuis, J. M. Yeomans, Lattice Boltzmann modelling of droplets on chemically heterogeneous surfaces, Future Generation Computer Systems 20 (6 SPEC. ISS.) (2004) 993-1001. doi:10.1016/j.future.2003.12.012.

[18] J. J. Huang, C. Shu, Y. T. Chew, Lattice Boltzmann study of bubble entrapment during droplet impact, International Journal for Numerical Methods in Fluids 65 (6) (2011) 655-682. doi:10.1002/fld.2209.

[19] S. Shen, F. Bi, Y. Guo, Simulation of droplets impact on curved surfaces with lattice Boltzmann method, International Journal of Heat and Mass Transfer 55 (23-24) (2012) 6938-6943. doi:10.1016/j.ijheatmasstransfer.2012.07.007.

[20] N. Moradi, F. Varnik, I. Steinbach, Roughness-gradientinduced spontaneous motion of droplets on hydrophobic surfaces: A lattice Boltzmann study, EPL (Europhysics Letters) 89 (2) (2010) 26006. doi:10.1209/0295-5075/89/26006. 
[21] L. Hao, P. Cheng, Lattice Boltzmann simulations of liquid droplet dynamic behavior on a hydrophobic surface of a gas flow channel, Journal of Power Sources 190 (2) (2009) 435-446. doi:10.1016/j.jpowsour.2009.01.029.

[22] A. Dupuis, J. M. Yeomans, Modeling droplets on superhydrophobic surfaces: Equilibrium states and transitions, Langmuir 21 (6) (2005) 26242629. doi:10.1021/la047348i.

[23] B. Peng, S. Wang, Z. Lan, W. Xu, R. Wen, X. Ma, Analysis of droplet jumping phenomenon with lattice Boltzmann simulation of droplet coalescence, Applied Physics Letters 102 (15) (2013) 151601. doi:10.1063/1.4799650.

[24] X. Liu, P. Cheng, X. Quan, Lattice Boltzmann simulations for selfpropelled jumping of droplets after coalescence on a superhydrophobic surface, International Journal of Heat and Mass Transfer 73 (2014) 195200. doi:10.1016/j.ijheatmasstransfer.2014.01.060.

[25] Z. Yu, O. Hemminger, L.-S. Fan, Experiment and lattice Boltzmann simulation of two-phase gasliquid flows in microchannels, Chemical Engineering Science 62 (24) (2007) 7172-7183. doi:10.1016/j.ces.2007.08.075.

[26] X. Frank, D. Funfschilling, N. Midoux, H. Z. Li, Bubbles in a viscous liquid: lattice Boltzmann simulation and experimental validation, Journal of Fluid Mechanics 546 (2005) 113-122. doi:10.1017/S0022112005007135.

[27] Y. Tanaka, Y. Washio, M. Yoshino, T. Hirata, Numerical simulation of dynamic behavior of droplet on solid surface by the two-phase lattice Boltzmann method, Computers \& Fluids 40 (1) (2011) 68-78. doi:10.1016/j.compfluid.2010.08.007.

[28] F. Varnik, M. Gross, N. Moradi, G. Zikos, P. Uhlmann, P. MüllerBuschbaum, D. Magerl, D. Raabe, I. Steinbach, M. Stamm, Stability and dynamics of droplets on patterned substrates: insights from experiments and lattice Boltzmann simulations., Journal of physics. Condensed matter : an Institute of Physics journal 23 (18) (2011) 184112. doi:10.1088/0953-8984/23/18/184112. 
[29] S. Wannaborworn, M. R. Mackley, Y. Renardy, Experimental observation and matching numerical simulation for the deformation and breakup of immiscible drops in oscillatory shear, Journal of Rheology 46 (5) (2002) 1279-1293. doi:10.1122/1.1501960.

[30] S. Guido, F. Greco, Dynamics of a Liquid Drop in a Flowing Immiscible Liquid, Rheology Reviews 2004 (2004) 99-142.

[31] H. P. Jansen, K. Sotthewes, J. Van Swigchem, H. J. Zandvliet, E. S. Kooij, Lattice Boltzmann modeling of directional wetting: Comparing simulations to experiments, Physical Review E - Statistical, Nonlinear, and Soft Matter Physics 88 (1) (2013) 1-10. doi:10.1103/PhysRevE.88.013008.

[32] P. Ramachandran, G. Varoquaux, Mayavi: 3D visualization of scientific data, Computing in Science and Engineering 13 (2) (2011) 40-51. arXiv:arXiv:1010.4891v1, doi:10.1109/MCSE.2011.35.

[33] W. Streule, T. Lindemann, G. Birkle, R. Zengerle, P. Koltay, PipeJet: A Simple Disposable Dispenser for the Nano- and Microliter Range, Journal of Laboratory Automation 9 (5) (2004) 300-306. doi:10.1016/j.jala.2004.08.008.

[34] L. Riegger, M. M. Mielnik, A. Gulliksen, D. Mark, J. Steigert, S. Lutz, M. Clad, R. Zengerle, P. Koltay, J. Hoffmann, Dye-based coatings for hydrophobic valves and their application to polymer labs-on-a-chip, Journal of Micromechanics and Microengineering 20 (4) (2010) 045021. doi:10.1088/0960-1317/20/4/045021.

[35] X. Shan, H. Chen, Lattice Boltzmann model for simulating flows with multiple phases and components, Physical Review E 47 (3) (1993) 18151819. doi:10.1103/PhysRevE.47.1815.

[36] R. Benzi, L. Biferale, M. Sbragaglia, S. Succi, F. Toschi, Mesoscopic modeling of a two-phase flow in the presence of boundaries: The contact angle, Physical Review E 74 (2) (2006) 021509. doi:10.1103/PhysRevE.74.021509.

[37] S. Chen, G. D. Doolen, Lattice Boltzmann Method For Fluid Flows, Annual Review of Fluid Mechanics 30 (1) (1998) 329-364. doi:10.1146/annurev.fluid.30.1.329. 
[38] C. K. Aidun, J. R. Clausen, Lattice-Boltzmann Method for Complex Flows, Annual Review of Fluid Mechanics 42 (1) (2010) 439-472. doi:10.1146/annurev-fluid-121108-145519.

[39] S. N. Sinha, M. Pollefeys, Synchronization and calibration of camera networks from silhouettes, Proceedings - International Conference on Pattern Recognition 1 (2004) 116-119. doi:10.1109/ICPR.2004.1334021.

[40] Y. Caspi, D. Simakov, M. Irani, Feature-based sequence-to-sequence matching, International Journal of Computer Vision 68 (1) (2006) 5364. doi:10.1007/s11263-005-4842-z.

[41] Rao, Gritai, Shah, Syeda-Mahmood, View-invariant alignment and matching of video sequences, Proceedings Ninth IEEE International Conference on Computer Vision (2003) 939-945 vol.2doi:10.1109/ICCV.2003.1238449.

[42] C. Lu, M. Mandal, A robust technique for motion-based video sequences temporal alignment, IEEE Transactions on Multimedia 15 (1) (2013) 70-82. doi:10.1109/TMM.2012.2225036.

[43] D. Perperidis, R. H. Mohiaddin, D. Rueckert, Spatio-temporal free-form registration of cardiac MR image sequences, Medical Image Analysis 9 ( 5 SPEC. ISS.) (2005) 441-456. doi:10.1016/j.media.2005.05.004.

[44] M. d. C. Valdés Hernández, P. J. Gallacher, M. E. Bastin, N. A. Royle, S. M. Maniega, I. J. Deary, J. M. Wardlaw, Automatic segmentation of brain white matter and white matter lesions in normal aging: Comparison of five multispectral techniques, Magnetic Resonance Imaging 30 (2) (2012) 222-229. doi:10.1016/j.mri.2011.09.016.

[45] K. McGuinness, N. E. O'Connor, A comparative evaluation of interactive segmentation algorithms, Pattern Recognition 43 (2) (2010) 434444. doi:10.1016/j.patcog.2009.03.008.

[46] A. R. S. Marcal, A. S. Rodrigues, A method for multi-spectral image segmentation evaluation based on synthetic images, Computers and Geosciences 35 (8) (2009) 1574-1581. doi:10.1016/j.cageo.2008.11.008. 
[47] J. Hyväluoma, A. Koponen, P. Raiskinmäki, J. Timonen, Droplets on inclined rough surfaces, The European Physical Journal E 23 (3) (2007) 289-293. doi:10.1140/epje/i2007-10190-7.

[48] A. Mazloomi Moqaddam, S. S. Chikatamarla, I. V. Karlin, Simulation of Droplets Collisions Using Two-Phase Entropic Lattice Boltzmann Method, Journal of Statistical Physics 161 (6) (2015) 1420-1433. doi:10.1007/s10955-015-1329-3.

[49] D.-J. Huang, T.-S. Leu, Condensation heat transfer enhancement by surface modification on a monolithic copper heat sink, Applied Thermal Engineering 75 (2015) 908-917. doi:10.1016/j.applthermaleng.2014.10.019.

[50] R. G. Chaudhuri, S. Paria, Dynamic contact angles on PTFE surface by aqueous surfactant solution in the absence and presence of electrolytes, Journal of Colloid and Interface Science 337 (2) (2009) 555-562. doi:10.1016/j.jcis.2009.05.033.

[51] I. V. Roisman, L. Opfer, C. Tropea, M. Raessi, J. Mostaghimi, S. Chandra, Drop impact onto a dry surface: Role of the dynamic contact angle, Colloids and Surfaces A: Physicochemical and Engineering Aspects 322 (1-3) (2008) 183-191. doi:10.1016/j.colsurfa.2008.03.005.

[52] C. W. Visser, P. E. Frommhold, S. Wildeman, R. Mettin, D. Lohse, C. Sun, Dynamics of high-speed micro-drop impact: numerical simulations and experiments at frame-to-frame times below 100 ns, Soft Matter 11 (9) (2015) 1708-1722. doi:10.1039/C4SM02474E. 

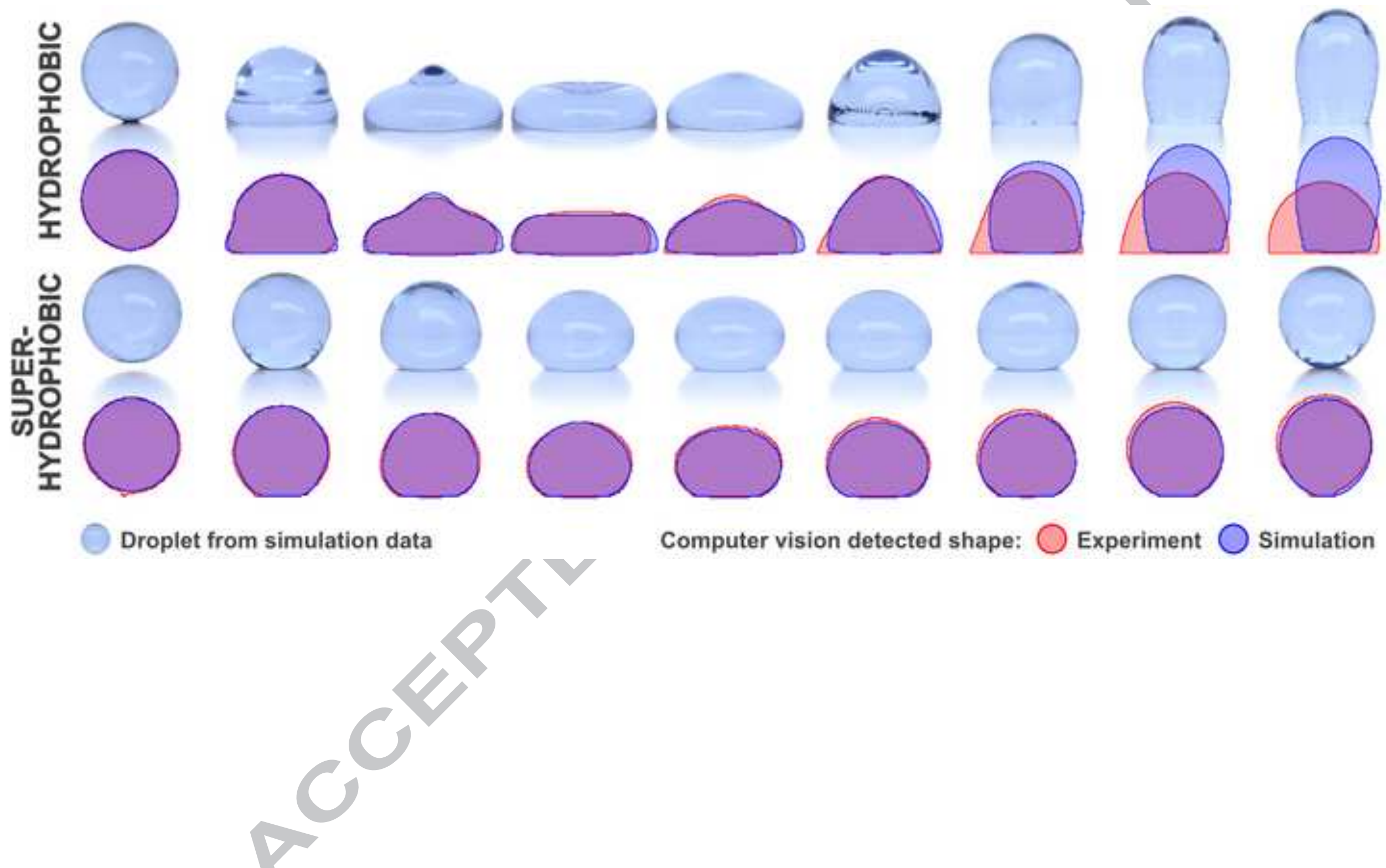

Computer vision detected shape: $\bigcirc$ Experiment $\bigcirc$ simulation 